\title{
Analysis on Different Techniques Used For Sentimental Analysis
}

\author{
Praveen Kulkarni ${ }^{1}$, Dr. Rajesh T.M ${ }^{2}$, \\ Assistant Professor CSE Department, Christ( Deemed to be University) Bangalore, \\ Karnataka, India \\ Assistant Professor CSE Department, Dayananda Sagar University Bangalore, \\ Karnataka, India \\ Email: Praveen.kulkarni@christuniversit.in ${ }^{1}$,rajesh-cse@dsu.edu.in ${ }^{2}$
}

\begin{abstract}
In the resent years use of pictures to precise points of read, opinions, emotions and feelings has increased tremendously on social platforms like facebook ,Instagram ,Flickr, Twitter etc. As we have a tendency to understand an image than talking thousand words about anything .The analysis of the emotions within the pictures generated by the user is more and more vital to develop completely different applications. A good deal of analysis has been done to research the feeling of human matter knowledge. There's an awfully restricted work that focuses on the sentimental analysis of image knowledge. During this role, we have a tendency to study the matter of understanding human feelings in large-scale social network pictures, taking into consideration each visual content and discourse data. The challenge lies within the linguistics gap between low-level visual characteristics and feelings of a better level image.Our review makes use of the link between the visual and therefore the relevant message to search out the discourse gap within the feelings of the image.
\end{abstract}

Index Terms- sentimental analysis, lexicon based, machine learning based, hybrid approach

\section{INTRODUCTION}

Recently, users in social media are more and more using private pictures and Video for expressing their feelings; opinions and can contribute to their experiences within the media. Such a study on visual sentiments on an outsized scale with content envisioned will facilitate US to extract the sensation of users thereon events or themes. The prediction of the sensation from the visual content is complementary to the analysis of the sentiments. Public from completely different background can perceive with no difficulty the content in a picture or a video. Additionally to nice simple Visual content out there, current IT communications is additionally loads cheaper and a lot of powerful to try to machine study of complete visual content. The analysis of the feeling of such visual content on an outsized scale will facilitate to higher extract the emotions of users towards actions or topic, alike those of tweets within the image, in order that the forecast of the feeling of image content is like the analysis of matter feeling.

Automatic logical thinking of sentimental analysis is increasing and therefore the variety of user-generated photos is changing into more and more vital for several applications within the fields of health, social science, communication studies, selling and plenty of laptop sub-areas, like artificial vision. Great variety of strategies and therefore the techniques with enhancements are planned for the sentimental analysis downside in numerous level and task. The most aim of the survey is classify Sentimental analysis techniques normally and additionally the centre of interest is on a definite echelon or activity.

In this analysis, we tend to study the analysis of feelings while not supervising for pictures of social networks with matter info through analysis. Interconnected challenge is that 1 st method model interaction between systems and matter knowledge during a systematic way for support sentiment prediction mistreatment each sources of data, and another major challenge is a way to use matter knowledge to facilitate unattended Sentiment study for social network pictures. And additionally review the most analysis issues conferred during this field

\section{METHODS USED IN SENTIMENTAL ANALYSIS}

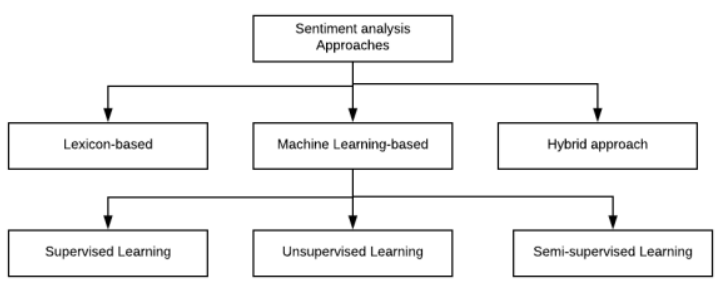

Figure 1: Techniques and Approaches of Sentimental Analysis 


\section{Available online at www.ijrat.org}

Machine learning is a technique of approach and additionally tongues process, lexical resources and additionally hybrid approaches area unit a number of the approaches. The approach supported lexical depends on the analysis of the vocabulary of opinion accustomed analyze the text. There are unit 2 strategies during this approach. The dictionary-based approach, that consists find opinions so looking the lexicon for its synonyms and antonyms. The corpusbased come back within sight of list of opinion so appearance different opinions. This might be completed mistreatment either applied math or the linguistics strategies. Various techniques and approaches of sentimental analysis as shown in Figure 1 are been discussed here.

The machine learning approach includes each the Support Vector Machine (SVM) and also the Naïve theorem classification for analysis [5]. SVM could be a supervised learning model that's primarily accustomed examine knowledge and build a distinction knowledge models which will be used for regression and classification analysis [3].

The naive theorem classification is principally supported the Naïve-Bayes theorem. Use the ideas of upper likelihood and also the theorem risk [6]. NLPbased approaches and lexical resources or lexicalbased approaches for the most part use components of vocal data and WorldNet [7]. There are a unit several approaches that area unit enforced by combining alleged hybrid approaches that close with machine learning and lexical resource [8].

Basic plan of machine learning, Suppose we've some entries $\mathrm{P} 1, \mathrm{P} 2, \ldots . \mathrm{Pn}$ and also the various objectives Q1, Q2, ... Qn. I know, coaching examples area unit a collection of level data: In the in the meantime, it's probable $(\mathrm{Q} / \mathrm{P})$.Unsupervised learning is incredibly a lot of associated with the matter of density assessment within the statistics. A collection of entries within the variety of new things area unit used for unsupervised learning. In likelihood notation it's known as joint likelihood or probability $(\mathrm{P}, \mathrm{Q})$.

\subsection{Supervised Learning}

Supervised learning because the name essentially, supervised learning may be a learning during which we tend to teach or train the machine victimization knowledge that's well tagged, which suggests we tend to area unit already tagged with the proper answer. And this can be given a brand new set of examples (data). Supervised learning issues area unit classified as "regression" and "classification" issues. In a very regression downside, we tend to are attempting to predict the results among

\subsection{Unsupervised Learning}

Study Image Sentiment Analysis practice more and more Trained and Domain Transferred Deep Networks by Quanzeng You and Jiebo Nilotic Hailin Jin and Jianchao principle. Throughout this work, we've got a bent to first designed Associate in nursing applicable CNN. Architecture for the analysis of the sensation of the image. We have a tendency to get partially $1,000,000$ exercise samples by means that of a basic feeling algorithmic rule for labeling pictures in flickr. To use this labeled knowledge of thunderous machines, we have a tendency to use a progressive strategy to refine the subterranean network. Moreover, we have a tendency to retrieve the concert of pictures in twitter by inducement shift of Twitter. We've got extended Experiments on Twitter pictures labeled manually. CNN can do higher results Performance within the analysis of the sensation of pictures those competition algorithms

\subsection{Semi Supervised Learning.}

Semi supervised learning with active knowledge can be increased through additional objectives through interactive consultations by the trainers, who were not available during the initial training phase. This is generalized through proactive learning and is also called optimal experimental design. Semi-supervised learning in-between the supervised and unsupervised. Qualified human experts to do it in many practical situations. Therefore, in the absence of semisupervised algorithms are the best candidates for the construction of the model. These methods take advantage of the idea that even if belonging to unlabeled data sets is unknown. The main plan behind SSL is that, even though they do not contain any class information according to [2]. Therefore, when SSL is not labeled, data can be improved compared to supervised learning. Furthermore, SSL does not restrict learning of unsupervised approach is, if we include some forms of past knowledge into the models of unsupervised.

\section{RELATED WORK}

Predefined dictionaries to measure the sensitivity level of tweets were used in 2010 and 2011. Furthermore, Hu et al. (2013) have included social cues in their analysis of emotions without supervision reference framework. They defined and integrated the indication of emotions and relationships in a framework to learn the parameters for their classifier of emotions.

There also are quite few modern works on visual sentiment analysis. Siersdorfer et al. in 2010 propose a machine learning algorithmic program for estimating the sentiment of pictures by means that of constituent 


\section{E-ISSN: 2321-9637 \\ Available online at www.ijrat.org}

intensity facial look. motivated by the reality that sentiment involves high-level construct, which can be easier to expose by objects or attributes in pictures, each Borth et al. and Yuan et al.[9] 2013 supply to require up visual entities or attributes as options meant for visual sentiment study.

Sentiment Analysis for societal medium Images [10] In this suggestion, we study the difficulty of understanding the individual feelings of a significant group of Internet descriptions based on commonly images Contextual features and information of social networks. Despite the excellent feeling in analyzing the user's feeling based on textual information, the analysis of the feeling behind the image, the content has been for the most part ignored.The challenge of the highest level of prediction of the feelings underlying the images.

A Deep Visual-Semantic Embedding Model [11] In this work, they present a new semantic visual depth. The image is displayed using image data with both label and semantic information obtained from nonannotated text. They show that this model corresponds to the most modern performances in the challenge of recognizing the Image Net objects of the 1000 class while creating additional semantically cheap mistakes, and that they additionally show that linguistics data will be exploited to create forecasts on tens of thousands of pictures. Tags not ascertained throughout coaching. Linguistics information improves these zero-shot forecasts by achieving success rates up to eighteen

How do your friends on social media disclose your emotions?[12] In this document they formally formulate the problem and propose a new way to learn emotions by modeling together images published by public users and observations additional to their associates. We can distinguish those comments that are closely associated with emotions. We can distinguish those comments that are closely associated with emotions. Expression for a picture of the opposite are irrelevant. Experiments in associate open Flickr information set show that the planned model will improve $+37.4 \%$ of $F 1$ the accuracy to deduce user emotions. Additional Interactions between $1.0 \%$ of the foremost overpriced friends.

Robust Image Sentiment Analysis Using Progressively Trained and Domain Transferred Deep Networks[13].In this work, we first designed an appropriate CNN. Architecture for the analysis of the feeling of the image. We get partly a million exercise samples by means of a basic feeling Algorithm for labeling images in flickr. To use this tagged data of earsplitting machines, we use a progressive strategy to refine the subterranean network. Moreover, we get better the concert of images in twitter by inducing shift of Twitter. We have extended Experiments on Twitter images tagged manually. CNN can achieve better results Performance in the analysis of the feeling of images that competition algorithms.

Visual Sentiment Analysis for Social pictures exploitation Transfer Learning Approach [14] during this paper, they counsel a clever visual sentiment analysis framework exploitation transfer learning approach to predict sentiment. we tend to build use of hyper parameters well-read from a awfully deep convolution neural network to initialize our network illustration to place off over fitting

\section{CONCLUSION}

The list surveys are wont to maximize the potency of the technology we have a bent to mention and also the method used. While the sector of opinion is new, there are many strategies offered to supply completely different levels of presidency, national security. During this document we have a leaning to classify some recent articles given within the Storm Troops field supported their techniques. We have a tendency to found machine-based techniques that embrace supervised learning, semi-supervised learning techniques, Lexicon-based techniques, and hybrid techniques. It doesn't target any level or specific task in Sentimental analysis. It's an honest supply for beginners World Health Organization don't have any background during this field. There should be the simplest way to match these techniques in completely different tasks at different levels. As a result of the character of the information set used was completely different, the prevailing analysis of the various strategies are used. In general, productive techniques are probably to be an honest integration of hybrid approaches and tongue process techniques. Open issues are operating in several domains; The classification of feelings supported deficient labeled information remains a difficult issue; there's an absence of analysis in alternative languages besides English; and also the existing techniques are still offered to treat advanced sentences that need over a sense of words and an easy analysis.

\section{REFERENCES}

[1] Yilin Wang, SuhangWang, Jiliang Tang, Huan Liu, Baoxin Li :Unsupervised Sentiment Analysis for Social Media Images

[2] B. Liu(2012),: Sentiment analysis and opinion mining, Synthesis Lectures on Human Language Technologies, vol. 5, pp. 1-167 


\section{Available online at www.ijrat.org}

[3] A. L. Maas, R. E. Daly, P. T. Pham, D. Huang, A. Y. Ng, and C. Potts,(2011) :Learning word vectors for sentiment analysis, in Proceedings of the 49th Annual Meeting of the Association for Computational Linguistics: Human Language Technologies-Volume 1, , pp. 142-150.

[4] C. D. Manning and H. Schutze:(1999) Foundations of statistical natural language processing. MIT Press.

[5] X. Hu, L. Tang, J. Tang, and H. Liu,(2013) :Exploiting social relations for sentiment analysis in microblogging,,in Proceedings of the sixth ACM international conference on Web search and data mining, , pp. 537-546.

[6]M. K. Dalal and M. A. Zaveri,(2014) :Opinion Mining from Online User Reviews Using Fuzzy Linguistic Hedges,Applied Computational Intelligence and Soft Computing, vol, p. 9, 2014.

[7]E. Cambria, C. Havasi, and A. Hussain,(2012) :SenticNet 2: A Semantic and Affective Resource for Opinion Mining and Sentiment Analysis, in FLAIRS Conference, , pp. 202-207.

[8]A. Ortigosa, J. M. Martín, and R. M. Carro, (2014)Sentiment analysis in Facebook and its application to e-learning,Computers in Human Behavior, vol. 31, pp. 527-541.

[9]Robust Image Sentiment Analysis Using Progressively Trained and Domain Transferred Deep Networks(2014) Quanzeng You, Jiebo Luo, Hailin Jin, Jianchao Yang

[10] Yilin Wang and Baoxin Li "Sentiment Analysis for Social Media Images."

[11] A. Frome, G. S. Corrado, J. Shlens, S. Bengio, J. Dean, M. A. Ranzato, and T. Mikolov, (2013) DeViSE: A deep visual-semantic embedding model, in Proc. Advances in Neural Information Processing Systems (NIPS), pp. 2121-2129.

[12] Y. Yang, J. Jia, S. Zhang, B. Wu, Q. Chen, J. Li, and J. Tang,(2014) :How do your friends on social media disclose your emotions? in Proc. AAAI Conf. Artificial Intelligence (AAAI), 2014, pp. 306-312.

[13]Q. You, J. Luo, H. Jin, and J. Yang, :Robust(2015) image sentiment analysis using progressively trained and domain transferred deep networks.

[14]Jyoti Islam ,Yanqing Zhang(2016) :Visual Sentiment Analysis for Social Images Using Transfer Learning Approach, IEEE International Conferences on Big Data and Cloud Computing . 